NDA-FP-61

May 1999

\title{
Thermodynamics of Schwarzschild-(Anti-)de Sitter Black Holes with account of quantum corrections
}

\author{
SHIN'ICHI NOJIRI \\ Department of Mathematics and Physics \\ National Defence Academy \\ Hashirimizu Yokosuka 239, JAPAN \\ A Tomsk Pedagogical University \\ 634041 Tomsk, RUSSIA
}

\begin{abstract}
We discuss the quantum corrections to thermodynamics (and geometry) of S(A)dS BHs using large $N$ one-loop anomaly induced effective action for dilaton coupled matter (scalars and spinors). It is found the temperature, mass and entropy with account of quantum effects for multiply horizon SdS $\mathrm{BH}$ and SAdS BH what also gives the corresponding expressions for their limits: Schwarzschild and de Sitter spaces. In the last case one can talk about quantum correction to entropy of expanding Universe.

The anomaly induced action under discussion corresponds to $4 \mathrm{~d}$ formulation ( $s$-wave approximation, $4 \mathrm{~d}$ quantum matter is minimal one) as well as $2 \mathrm{~d}$ formulation (complete effective action, $2 \mathrm{~d}$ quantum matter is dilaton coupled one). Hence, most of results are given for the same gravitational background with interpretation as $4 \mathrm{~d}$ quantum corrected $\mathrm{BH}$ or $2 \mathrm{~d}$ quantum corrected dilatonic BH. Quantum aspects of thermodynamics of $4 \mathrm{~d}$ 't Hooft $\mathrm{BH}$ model are also considered.

\footnotetext{
${ }^{1}$ e-mail : nojiri@cc.nda.ac.jp

2 e-mail : odintsov@mail.tomsknet.ru, sergei.odintsov@itp.uni-leipzig.de
} 


\section{Introduction}

The famous Bekenstein-Hawking entropy of black hole [2] is known to be proportional to the surface area of its event horizon. It gives the bright manifestation of analogy between thermodynamics and BHs [1]. However, despite numerous attempts varying from strings [3], three dimensional gravity [由, 5], induced gravity [6], etc., the derivation of BH entropy from statistical mechanics is not yet completely clear.

Among other (thermodynamic) quantities used to describe BHs one can mention not only entropy but also temperature, horizon radius, mass, energy, charges,etc. In order to understand better the thermodynamic properties of $\mathrm{BHs}$ it is extremely important to find the unified way to evaluate the quantum corrections to all above characteristics of BH. In its own turn, such study is expected to give the insights to the better formulations of quantum gravity.

Unfortunately, the calculation of quantum corrections to thermodynamics of different BHs is not easy task. Usual approach is to consider (simple) quantum matter on some BH background (as a rule Rindler space) where spectrum of the corresponding operator is known. Then, in frames of some regularization one can find the effective action (or stress energy tensor) what may be used as quantum correction to classical action. This very tedious procedure should be repeated again for each specific geometry (when it is possible). The complete effective action (EA) on an arbitrary gravitational background is not yet known (see, for a review [11]). It would be really interesting to present more universal approach, at least in the situations when EA is known for some classes of backgrounds.

In the present paper we suggest such universal prescription to the calculation of quantum corrections to BHs. It is based on anomaly induced EA for $2 \mathrm{~d}$ dilaton coupled matter (scalars and spinors). Such EA is found on an arbitrary dilaton-gravitational background. From $4 \mathrm{~d}$ point of view it gives so-called s-wave approximation EA and from $2 \mathrm{~d}$ point of view it gives total one-loop EA for dilaton coupled matter which is typical in 2d Brans-Dickematter theory (in Einstein frame). As dilaton and metric dependence of this EA is known it may be easily added to classical action. After that one is left with modified but still CLASSICAL gravity where calculation of thermodynamics of BHs is now routine work. Moreover, the results obtained in such way have $4 \mathrm{~d}$ interpretation as well as $2 \mathrm{~d}(4 \mathrm{~d} \mathrm{BH}$ may be easily interpreted as $2 \mathrm{~d}$ dilatonic $\mathrm{BH})$. 
We mainly discuss multiply horizon Schwarzschild-(Anti-)de Sitter $(\mathrm{S}(\mathrm{A}) \mathrm{dS}) \mathrm{BHs}$ which typically may occur in the early Universe as primordial ones. In addition, as limiting backgrounds they have de Sitter and Schwarzschild spaces. Note that S(A)dS BHs do not normally appear at the final stage of star collapse. Nevertheless, still there maybe mechanisms to produce them: via $\mathrm{BHs}$ creation at the early Universe [23] or via direct inducing of such primordial objects due to quantum effects [24.

They also may demonstrate the realization of beautiful BHs antievaporation process [13, 25] which may become the basis for creation of muliply inflationary Universes [13, 24]. It is important that anti-evaporation of BHs may put cosmological limits to the content of GUTs 25.

The paper is organised as follows. The review of anomaly induced EA evaluation and construction of effective equations of motion is presented in the next section. In section three, using such EA we find quantum corrections to $\mathrm{BH}$ entropy, mass, horizon radius and temperature for $4 \mathrm{~d}$ and $2 \mathrm{~d}$ Schwarzschild BHs. In section 4 the same problem is solved for SdS (or SAdS) BHs. The results of previous section may be reproduced from such calculation by taking the correspondent limit. Quantum properties of another limit (de Sitter space) are also discussed. In this case we get the quantum correction to the entropy of expanding Universe. In the last section we discuss quantum corrections to thermodynamics of 't Hooft $\mathrm{BH}$ model, working in the same fashion. Some outlook related with quite general character of our approach as well as the possibility of other applications is given in final section.

\section{The effective action and equations of mo- tion}

We will start from the action of Einstein gravity with $N$ minimal real scalars and $M$ Majorana fermions

$$
\begin{aligned}
S_{4 d}= & -\frac{1}{16 \pi G} \int d^{4} x \sqrt{-g_{(4)}}\left(R^{(4)}-2 \Lambda\right) \\
& +\int d^{4} x \sqrt{-g_{(4)}}\left(\frac{1}{2} \sum_{i=1}^{N} g_{(4)}^{\alpha \beta} \partial_{\alpha} \chi_{i} \partial_{\beta} \chi_{i}+\sum_{i=1}^{M} \bar{\psi}_{i} \gamma^{\mu} \nabla_{\mu} \psi_{i}\right)
\end{aligned}
$$


where $\chi_{i}$ and $\psi_{i}$ are real scalars and Majorana spinors, respectively. In order to apply large $N$ approach, $N$ and $M$ are considered to be large, $N, M \gg 1$, $G$ and $\Lambda$ are gravitational and cosmological constants, respectively.

The convenient choice for the spherically symmetric spacetime is the following one

$$
d s^{2}=g_{\mu \nu} d x^{\mu} d x^{\nu}+e^{-2 \phi} d \Omega,
$$

where $\mu, \nu=0,1, g_{\mu \nu}$ and $\phi$ depend only on $x^{0}, x^{1}$ and $d \Omega$ corresponds to the two-dimensional sphere.

The action (1), reduced according to (2) takes the form

$$
\begin{aligned}
S_{\text {red }}= & \int d^{2} x \sqrt{-g} \mathrm{e}^{-2 \phi}\left[-\frac{1}{16 \pi G}\left\{R+2(\nabla \phi)^{2}-2 \Lambda+2 \mathrm{e}^{2 \phi}\right\}\right. \\
& \left.+\frac{1}{2} \sum_{i=1}^{N}\left(\nabla \chi_{i}\right)^{2}+\sum_{i=1}^{2 M} \bar{\psi}_{i} \gamma^{\mu} \nabla_{\mu} \psi_{i}\right]
\end{aligned}
$$

Note that the fermion degrees of freedom after reduction are twice of original ones.

Working in large $N$ and $s$-wave approximation, one can calculate the quantum correction to $S_{\text {red }}$ (effective action). Using $2 \mathrm{~d}$ conformal anomaly for dilaton coupled scalar, calculated in [7] (see also [8, 9, [0]) one can find the anomaly induced effective action [8, 9] (with accuracy up to conformally invariant functional for the total effective action, see [11] for a review). There is no consistent approach to calculate this conformally invariant functional in closed form. However, one can find this functional as some expansion of Schwinger-DeWitt type [12 keeping only the leading term. Then, the effective action may be written in the following form [9, 12] (these works were related with only scalars)

$$
\begin{aligned}
W= & -\frac{1}{8 \pi} \int d^{2} x \sqrt{-g}\left[\frac{N+M}{12} R \frac{1}{\Delta} R-N \nabla^{\lambda} \phi \nabla_{\lambda} \phi \frac{1}{\Delta} R\right. \\
& \left.+\left(N+\frac{2 M}{3}\right) \phi R+2 N \ln \mu_{0}^{2} \nabla^{\lambda} \phi \nabla_{\lambda} \phi\right] .
\end{aligned}
$$

where $\Delta$ is two-dimensional laplacian, $\mu_{0}^{2}$ is a dimensional parameter. Here, the first term represents the Polyakov anomaly induced action, the second and third terms give the dilaton dependent corrections to the anomaly induced action The last term (conformally invariant functional) is found in 
ref. 112]. Note that EA for dilaton coupled spinor has been found in ref. [17] where it was shown that unlike to scalar case there is no ambiguety in EA related with conformally invariant functional. Spinor EA is known exactly in both cases: minimal or dilaton coupled spinor.

Working in the conformal gauge

$$
g_{ \pm \mp}=-\frac{1}{2} \mathrm{e}^{2 \rho}, \quad g_{ \pm \pm}=0
$$

the equations of motion may be obtained by the variation of $\Gamma=S_{\text {red }}+W$ with respect to $g^{ \pm \pm}, g^{ \pm \mp}$ and $\phi$

$$
\begin{aligned}
0= & \frac{\mathrm{e}^{-2 \phi}}{4 G}\left(2 \partial_{r} \rho \partial_{r} \phi+\left(\partial_{r} \phi\right)^{2}-\partial_{r}^{2} \phi\right) \\
& -\frac{N+M}{12}\left(\partial_{r}^{2} \rho-\left(\partial_{r} \rho\right)^{2}\right)-\frac{N}{2}\left(\rho+\frac{1}{2}\right)\left(\partial_{r} \phi\right)^{2} \\
& -\frac{N+\frac{2 M}{3}}{4}\left(2 \partial_{r} \rho \partial_{r} \phi-\partial_{r}^{2} \phi\right)-\frac{N}{4} \ln \mu_{0}^{2}\left(\partial_{r} \phi\right)^{2}+N t_{0} \\
0= & \frac{\mathrm{e}^{-2 \phi}}{8 G}\left(2 \partial_{r}^{2} \phi-4\left(\partial_{r} \phi\right)^{2}-2 \Lambda \mathrm{e}^{2 \rho}+2 \mathrm{e}^{2 \rho+2 \phi}\right) \\
& +\frac{N+M}{12} \partial_{r}^{2} \rho+\frac{N}{4}\left(\partial_{r} \phi\right)^{2}-\frac{N+\frac{2 M}{3}}{4} \partial_{r}^{2} \phi \\
0 & -\frac{\mathrm{e}^{-2 \phi}}{4 G}\left(-\partial_{r}^{2} \phi+\left(\partial_{r} \phi\right)^{2}+\partial_{r}^{2} \rho+\Lambda \mathrm{e}^{2 \rho}\right) \\
& +\frac{N}{2} \partial_{r}\left(\rho \partial_{r} \phi\right)+\frac{N+\frac{2 M}{3}}{4} \partial_{r}^{2} \rho+\frac{N}{2} \ln \mu_{0}^{2} \partial_{r}^{2} \phi .
\end{aligned}
$$

Here, $t_{0}$ is a constant which is determined by the initial conditions. Below we are interested in the static solution that is why we replace $\partial_{ \pm} \rightarrow \pm \frac{1}{2} \partial_{r}$ where $r$ is radial coordinate.

Furthermore, we change the radial coordinate $r$ by the new coordinate $x$

$$
x=\mathrm{e}^{-\phi},
$$

which corresponds to the usual coordinate choice in the Schwarzschild metric:

$$
\begin{aligned}
d s^{2} & =-\mathrm{e}^{2 \rho} d t^{2}+\mathrm{e}^{2 \sigma} d x^{2}+x^{2} d \Omega^{2} \\
\mathrm{e}^{\sigma} & =-\mathrm{e}^{\rho+\phi}\left(\frac{d \phi}{d r}\right)^{-1}
\end{aligned}
$$


Then we find

$$
\partial_{r}=\mathrm{e}^{\rho-\sigma} \partial_{x}, \quad \partial_{r}^{2}=\mathrm{e}^{2(\rho-\sigma)}\left(\partial_{x}^{2}+\left(\partial_{x} \rho-\partial_{x} \phi\right) \partial_{x}\right)
$$

and the equations (6), (7) and (8) can be rewritten as follows:

$$
\begin{aligned}
0= & -\frac{x}{4 G}\left(\partial_{x} \rho+\partial_{x} \phi\right)-\frac{N+M}{12}\left(\partial_{x}^{2} \rho-\partial_{x} \sigma \partial_{x} \rho\right)-\frac{N}{2 x^{2}}\left(\rho+\frac{a}{2}\right) \\
& +\frac{N+\frac{2 M}{3}}{4}\left(\partial_{x} \rho+\partial_{x} \phi\right)+N t_{0} \mathrm{e}^{2 \sigma-2 \rho} \\
0= & \frac{x^{2}}{4 G}\left(-\frac{1}{x}\left(\partial_{x} \rho-\partial_{x} \sigma\right)-\frac{1}{x^{2}}-\Lambda \mathrm{e}^{2 \sigma}+\frac{\mathrm{e}^{2 \sigma}}{x^{2}}\right) \\
& +\frac{N+M}{12}\left(\partial_{x}^{2} \rho+\left(\partial_{x} \rho\right)^{2}-\partial_{x} \sigma \partial_{x} \rho\right) \\
& +\frac{N+\frac{2 M}{3}}{4 x}\left(\partial_{x} \rho-\partial_{x} \sigma\right)-\frac{M}{6 x^{2}} \\
0= & -\frac{x^{2}}{4 G}\left(\frac{1}{x}\left(\partial_{x} \rho-\partial_{x} \sigma\right)+\partial_{x}^{2} \rho+\left(\partial_{x} \rho\right)^{2}-\partial_{x} \sigma \partial_{x} \rho+\Lambda \mathrm{e}^{2 \sigma}\right) \\
& +\frac{N}{4}\left\{-\frac{2}{x} \partial_{x} \rho+2 \rho\left(\frac{1}{x^{2}}-\frac{1}{x}\left(\partial_{x} \rho-\partial_{x} \sigma\right)\right)\right\} \\
& +\frac{N+\frac{2 M}{3}}{4}\left\{\partial_{x}^{2} \rho+\left(\partial_{r} \rho\right)^{2}-\partial_{x} \sigma \partial_{x} \rho\right\} \\
& +\frac{N a}{2}\left(\frac{1}{x^{2}}-\frac{1}{x}\left(\partial_{x} \rho-\partial_{x} \sigma\right)\right) .
\end{aligned}
$$

Here $a \equiv \ln \mu_{0}^{2}$. Combining (12) and (13), we obtain the following equation

$$
\begin{aligned}
0= & \frac{x^{2}}{4 G}\left(-\frac{1}{x^{2}}-\frac{2}{x} \partial_{x} \rho-\Lambda \mathrm{e}^{2 \sigma}+\frac{\mathrm{e}^{2 \sigma}}{x^{2}}\right) \\
& +\frac{N+M}{12}\left(\partial_{x} \rho\right)^{2}-\frac{N}{2 x^{2}} \rho+\frac{N+\frac{2 M}{3}}{2 x} \partial_{x} \rho-\frac{N a}{4 x^{2}} \mathrm{e}^{2 \rho}+N t_{0} \mathrm{e}^{2 \sigma-2 \rho}
\end{aligned}
$$

This last equation is necessary to delete the constant $t_{0}$ (which is defined by initial conditions) from further consideration. In the next sections we use above equations of motion in order to find quantum corrections to different $\mathrm{BH}$ configurations. Note also that there were also attempts in refs. [13, 9, 12, 14] to apply such EA (usually without logarithmic term and 
only for scalars) for quantum considerations around BHs (for semiclassical stress tensor with dilaton,see ref.[16]). Notice also that some terms of above EA have similarities with counterterms added by hands to CGHS dilatonic gravity model [18], forming its extension as RST model [19]. For a very incomplete list of references on the study of $2 \mathrm{~d}$ dilatonic $\mathrm{BHs}$ in these models, see refs. 20].

\section{Quantum corrections to 2d and 4d Schwarzschild black hole}

First we consider the case $\Lambda=0$. In the classical limit $(N \rightarrow 0)$, we obtain, of course, the Schwarzschild black hole as solution of equations of motion:

$$
\begin{aligned}
& \mathrm{e}^{2 \rho}=\mathrm{e}^{2 \rho_{0}}, \quad \mathrm{e}^{2 \sigma}=\mathrm{e}^{2 \sigma_{0}}, \\
& \mathrm{e}^{2 \rho_{0}}=\mathrm{e}^{-2 \sigma_{0}}=1-\frac{\mu}{x} .
\end{aligned}
$$

Here $\mu=2 G M_{\mathrm{BH}}$ and $M_{\mathrm{BH}}$ is the black hole mass. We now consider the quantum corrections by regarding $G N$ is small and assuming

$$
\rho=\rho_{0}+G N \Delta_{\rho}, \quad \sigma=\sigma_{0}+G N \Delta_{\sigma}\left(\sigma_{0}=-\rho_{0}\right) .
$$

Then substituting (17) into (12), we obtain

$$
\begin{aligned}
0= & -\frac{x}{4} \partial_{x}\left(\Delta_{\rho}+\Delta_{\sigma}\right)-\frac{A}{12}\left(\partial_{x}^{2} \rho_{0}-\partial_{x} \sigma_{0} \partial_{x} \rho_{0}\right) \\
& -\left(\frac{\rho_{0}}{2}+\frac{a+B-1}{4}\right) \frac{1}{x^{2}}+\frac{B}{4 x}\left(\partial_{x} \rho_{0}+\partial_{x} \sigma_{0}\right) \\
& +t_{0} \mathrm{e}^{2 \sigma_{0}-2 \rho_{0}}+\mathcal{O}(G N) .
\end{aligned}
$$

Substituting the classical solution (16) into (18), we obtain

$$
\begin{aligned}
0= & \partial_{x}\left(\Delta_{\rho}+\Delta_{\sigma}\right)+\frac{A}{3 x}\left\{-\frac{1}{4(x-\mu)^{2}}+\frac{3}{4 x^{2}}-\frac{1}{2 x(x-\mu)}\right\} \\
& +\{\ln (x-\mu)-\ln x+a+B-1\}-\frac{4 t_{0} x}{(x-\mu)^{2}}
\end{aligned}
$$

Here

$$
A \equiv \frac{N+M}{N}, \quad B \equiv \frac{N+\frac{2 M}{3}}{N}
$$


Eq.(19) can be easily integrated to give

$$
\begin{aligned}
& \Delta_{\rho}+\Delta_{\sigma} \\
& =A\left\{-\frac{1}{12 \mu(x-\mu)}+\frac{1}{12 \mu^{2}} \ln (x-\mu)\right. \\
& \left.+\frac{1}{8 x^{2}}+\frac{1}{6 \mu x}-\frac{1}{12 \mu^{2}} \ln \frac{x}{l}\right\} \\
& +\frac{a+B-1}{2 x^{2}}-\frac{1}{4 x^{2}}-\frac{1}{2 \mu x}+\frac{1}{2}\left(\frac{1}{x^{2}}-\frac{1}{\mu^{2}}\right) \ln \left(1-\frac{\mu}{x}\right) \\
& +4 t_{0}\left\{-\frac{\mu}{x-\mu}+\ln (x-\mu)\right\} .
\end{aligned}
$$

Here $l$ is a constant of the integration.

On the other hand, substituting (17) into (15), we get

$$
\begin{aligned}
0= & -\frac{x}{2} \partial_{x} \Delta_{\rho}+\frac{1}{2} \mathrm{e}^{2 \sigma_{0}} \Delta_{\sigma}+\frac{A}{12}\left(\partial_{x} \rho_{0}\right)^{2}-\frac{1}{2 x^{2}} \rho_{0} \\
& +\frac{B}{2 x} \partial_{x} \rho_{0}-\frac{a}{4 x^{2}}+t_{0} \mathrm{e}^{2 \sigma_{0}-2 \rho_{0}}+\mathcal{O}(G N) .
\end{aligned}
$$

Using (18) and (22), we can delete $\Delta_{\rho}$ and obtain

$$
\begin{aligned}
0= & \frac{x}{2} \partial_{x} \Delta_{\sigma}+\frac{1}{2} \mathrm{e}^{2 \sigma_{0}} \Delta_{\sigma} \\
& +\frac{A}{12}\left(2 \partial_{x}^{2} \rho_{0}-2 \partial_{x} \sigma_{0} \partial_{x} \rho_{0}+\left(\partial_{x} \rho_{0}\right)^{2}\right) \\
& +\left(\frac{\rho_{0}}{2}+\frac{a-B+1}{4}\right) \frac{1}{x^{2}} \\
& -\frac{B}{2 x} \partial_{x} \sigma_{0}-t_{0} \mathrm{e}^{2 \sigma_{0}-2 \rho_{0}}+\mathcal{O}(G N) .
\end{aligned}
$$

Substituting the classical solution (16) into (23), one gets

$$
\begin{aligned}
0= & \partial\left((x-\mu) \Delta_{\sigma}\right)+A\left\{-\frac{1}{24 x(x-\mu)}+\frac{1}{24 x^{2}}-\frac{7 \mu}{24 x^{3}}\right\} \\
+ & \frac{1}{2}\left(\frac{1}{x^{2}}-\frac{\mu}{x^{3}}\right)(a-B+1+\ln (x-\mu)-\ln x) \\
& +\frac{B \mu}{2 x^{3}}-\frac{2 t_{0} x}{x-\mu} .
\end{aligned}
$$


Integrating (24)

$$
\begin{aligned}
& \Delta_{\sigma}=\frac{1}{3(x-\mu)} \\
& \times\left\{\Delta_{S}+A\left(\frac{1}{8 \mu} \ln (x-\mu)-\frac{1}{8 \mu} \ln \frac{x}{l}+\frac{1}{8 x}-\frac{7 \mu}{16 x^{2}}\right)\right. \\
& +\frac{a-B+1}{2}\left(\frac{3}{x}-\frac{3 \mu}{2 x^{2}}\right) \\
& -\frac{3 B \mu}{4 x^{2}}-\frac{3 \mu}{4}\left(\frac{1}{x}-\frac{1}{\mu}\right)^{2} \ln \left(1-\frac{\mu}{x}\right)-\frac{3}{4 x}+\frac{3 \mu}{8 x^{2}} \\
& \left.+6 t_{0}(x+\mu \ln (x-\mu))\right\} .
\end{aligned}
$$

Here $\Delta_{S}$ is a constant of the integration.

Near the classical horizon $x \sim \mu, \Delta_{\sigma}$ in (25) behaves as

$$
\Delta_{\sigma} \sim \frac{1}{3(x-\mu)}\left[\left(\frac{A}{8 \mu}+6 t_{0} \mu\right) \ln (x-\mu)+\text { regular terms }\right] .
$$

The singularity coming from $\ln (x-\mu)$ vanishes if we choose

$$
t_{0}=-\frac{A}{48 \mu^{2}} .
$$

In the choice of (27), Eqs. (21) and (25) have the following forms:

$$
\begin{aligned}
& \Delta_{\rho}+\Delta_{\sigma} \\
& =A\left\{\frac{1}{8 x^{2}}+\frac{1}{6 \mu x}-\frac{1}{12 \mu^{2}} \ln \frac{x}{l}\right\} \\
& +\frac{a+B-1}{2 x^{2}}-\frac{1}{4 x^{2}}-\frac{1}{2 \mu x}+\frac{1}{2}\left(\frac{1}{x^{2}}-\frac{1}{\mu^{2}}\right) \ln \left(1-\frac{\mu}{x}\right), \\
& \Delta_{\sigma}=\frac{1}{3(x-\mu)} \\
& \times\left\{\Delta_{S}+A\left(-\frac{1}{8 \mu} \ln \frac{x}{l}+\frac{1}{8 x}-\frac{7 \mu}{16 x^{2}}\right)+\frac{a-B+1}{2}\left(\frac{3}{x}-\frac{3 \mu}{2 x^{2}}\right)\right. \\
& \left.-\frac{3 B \mu}{4 x^{2}}-\frac{3 \mu}{4}\left(\frac{1}{x}-\frac{1}{\mu}\right)^{2} \ln \left(1-\frac{\mu}{x}\right)-\frac{3}{4 x}+\frac{3 \mu}{8 x^{2}}+\frac{3 x}{2 \mu^{2}}\right\} .
\end{aligned}
$$

\footnotetext{
${ }^{3}$ This procedure tells that $t_{0}$ here corresponds to the constants $C$ and $D$ of the integration in [14].
} 
There are undetermined parameters $l$ and $\Delta_{S}$ coming from the constants of the integration. We now assume the radius $L$ of the universe is large $L \gg \mu$ but finite and we require

$$
\Delta_{\rho}=\Delta_{\sigma}=0 \quad \text { when } x=L .
$$

Then we find

$$
l=L, \quad \Delta_{S}=-\frac{3 L}{2 \mu^{2}} .
$$

Near the classical horizon, $\Delta_{\rho}+\Delta_{\sigma}$ is regular:

$$
\begin{aligned}
\Delta_{\rho}+\Delta_{\sigma} & \sim B_{S} \\
B_{S} & \equiv \frac{A}{\mu^{2}}\left(\frac{7}{24}-\frac{1}{12} \ln \frac{\mu}{l}\right)+\frac{a+B-1}{2 \mu^{2}}-\frac{3}{4 \mu^{2}}
\end{aligned}
$$

and $\Delta_{\sigma}$ behaves as

$$
\begin{aligned}
\Delta_{\sigma} & \sim \frac{C_{S}}{x-\mu} \\
C_{S} & \equiv-\frac{\Delta_{S}}{3}+\frac{A}{24 \mu} \ln \frac{\mu}{l}+\frac{5 A}{48 \mu}-\frac{a-B+1}{4 \mu}+\frac{B}{4 \mu}-\frac{3}{8 \mu} .
\end{aligned}
$$

Eqs.(31) and (32) tell that the scalar curvature, which is given by

$$
\begin{aligned}
R_{4} & =R+2(\nabla \phi)^{2}+2 \mathrm{e}^{2 \phi} \\
R & =-2 \mathrm{e}^{-2 \rho} \partial_{r}^{2} \rho \\
& =-2 \mathrm{e}^{-2 \sigma}\left(\partial_{x}^{2} \rho+\left(\partial_{x} \rho-\partial_{x} \sigma\right) \partial_{x} \rho\right),
\end{aligned}
$$

is regular when $x \sim \mu$. The horizon defined by $\mathrm{e}^{2 \rho}=0$, which corresponds to $x=\mu$ in the classical limit is given by

$$
\begin{aligned}
0 & =\mathrm{e}^{2 \rho} \\
& \sim \mathrm{e}^{2 \rho_{0}}\left(1+2 G N \Delta_{\rho}\right) \\
& \sim \frac{1}{\mu}\left(x-\mu+2 G N C_{S}\right) .
\end{aligned}
$$

Then the entropy, which is defined by the area of horizon divided by $4 G$, is given by

$$
S \sim \frac{\pi}{G}\left(x \mu-2 G N C_{S}\right)^{2}
$$




$$
\begin{aligned}
& \sim \frac{\pi \mu^{2}}{G}-4 \pi N C_{S} x \mu \\
& =\frac{\pi \mu^{2}}{G}-\pi N\left(-\frac{4 \mu \Delta_{S}}{3}+\frac{A}{6} \ln \frac{\mu}{l}+\frac{5 A}{12}-a+2 B-\frac{5}{2}\right) .
\end{aligned}
$$

The second term in (35) corresponds to the quantum correction. The equation (34) tells that the behavior of the metric near the horizon $x=\mu-2 G N C_{S}$ is given by

$$
\begin{aligned}
d s^{2} & =-\mathrm{e}^{2 \rho} d t^{2}+\mathrm{e}^{-2 \rho+2 G N\left(\Delta_{\rho}+\Delta_{\sigma}\right)} d x^{2}+x^{2} d \Omega^{2} \\
& \sim-\frac{x-\mu+2 G N C_{S}}{\mu} d t^{2}+\frac{\mu\left(1+2 G N B_{S}\right)}{x-\mu+2 G N C_{S}} d x^{2}+x^{2} d \Omega^{2} .
\end{aligned}
$$

Therefore the temperature $T$ (in the following, we put the Boltzmann constant $k$ to be unity, $k=1$ ) is given by

$$
\begin{aligned}
T & \sim \frac{1}{4 \pi \mu}\left\{1+G N\left(\frac{2 C_{S}}{\mu}-B_{S}\right)\right\} \\
& =\frac{1}{4 \pi \mu}\left[1+G N\left\{-\frac{2}{3 \mu} \Delta_{S}+\frac{A}{12 \mu^{2}}\left(-1+2 \ln \frac{\mu}{l}\right)-\frac{a}{\mu^{2}}+\frac{B}{2 \mu^{2}}\right\}\right]
\end{aligned}
$$

The second term corresponds to the quantum correction. Finally in this section, we consider the thermodynamical mass $E$, which is defined by

$$
d E=T d S
$$

Using the parameter $\mu$, (38) can be rewritten as

$$
\begin{aligned}
E & =\int d \mu T \frac{d S}{d \mu} \\
& =\frac{\mu}{2 G}+N\left(-\frac{L}{2 \mu^{2}}-\frac{A}{12 \mu} \ln \frac{\mu}{L}+\frac{a}{2 \mu}+\frac{B}{4 \mu}\right) .
\end{aligned}
$$

Here we used (30), (35) and (37). We also put $k=1$. The first term expresses the usual classical mass $M_{\mathrm{BH}}$ since $\mu=2 G M_{\mathrm{BH}}$ and the second term is due to the quantum effects. Notice the regularization scheme dependence vie the presence of parameter a in above expressions. It maybe fixed by the choice of physical regularization. 
The qualitative structures of the entropy (35), the temperature (37) and the energy (mass) (39) are similar to the corresponding quantities found in [14]. Note however that our result incorporates the quantum effects of not only scalars but also of spinors to BH configuration under consideration.

Let us turn now to $2 \mathrm{~d}$ formulation of above results. The classical black hole solution (16) can be regarded as a purely two dimensional object if we start with the reduced action (3). The thermodynamical quantities as the energy and entropy for the two dimensional charged black hole with dilaton are evaluated on the classical and one-loop levels in [12, 15]. In [15], the boundary of the universe is introduced at the radius $r=L$ as in (29). If there is a boundary, we need to add the boundary terms to the action in order that the variation with respect to the metric should be well-defined. By including the boundary term, the formula for the energy (mass) of the black hole with one-loop quantum correction was derived in [15] as follows,

$$
\begin{aligned}
E(\mu, L)= & -\frac{\mathrm{e}^{\lambda(L)}}{G} g^{\frac{1}{2}}(L) D^{\prime}(\phi(L)) \\
& -\frac{\hbar}{3} \mathrm{e}^{\lambda_{C L}(L)} g_{C L}^{-\frac{1}{2}}(L) g_{C L}^{\prime}(L)-\frac{c \hbar}{6} \mathrm{e}^{\lambda_{C L}(L)} g_{C L}^{\frac{1}{2}}(L) \phi^{\prime}(L) .
\end{aligned}
$$

Here $^{\prime}=\frac{d}{d x}$. The quantities appeared in (40) have the following correspondence with the quantities here,

$$
g(x)=\mathrm{e}^{2 \rho(x)}, \quad \mathrm{e}^{-\lambda(x)}, \quad D(\phi(x))=\frac{\mathrm{e}^{-2 \phi(x)}}{2}=\frac{x^{2}}{2} .
$$

Since only the quantum correction from the one scalar field was evaluated in [15], we need to do the following replacement

$$
\hbar \rightarrow N A, \quad c \rightarrow \frac{12 B}{A} .
$$

We should note that we usually choose $\hbar=1$ in this paper. In the expression (40), the quantities with suffices $C L$ correspond to the classical ones. If we choose the boundary condition as in (29), we find

$$
g(L)=g_{C L}(L)=1-\frac{\mu}{L}, \Lambda(L)=\Lambda_{C L}(L)=0 .
$$

Therefore

$$
E(\mu, L)=-\frac{L}{G}\left(1-\frac{\mu}{L}\right)^{\frac{1}{2}}-\frac{N A \mu}{3 L^{2}}\left(1-\frac{\mu}{L}\right)^{-\frac{1}{2}}+\frac{2 N B}{L}\left(1-\frac{\mu}{L}\right)^{\frac{1}{2}} .
$$


Since the energy (44) diverges in the limit of $L \rightarrow+\infty$, we need to subtract $E(0, L)$ before taking the limit of $L \rightarrow+\infty$. Then we obtain

$$
E_{\text {sub }} \equiv \lim _{L \rightarrow+\infty}(E(\mu, L)-E(0, L))=\frac{\mu}{2 G},
$$

which is nothing but the classical black hole mass. This tells that there is no the quantum correction for the mass or the boundary condition in (29) corresponds to the renormalization condition that the mass does not suffer the quantum correction. Even if the black hole is purely two dimensional one (but with dilaton), the definition of the temperature is not changed and one gets (37). Then using the energy (45), the temperature (37) and the definition of the entropy (38), we obtain the following expression of the entropy

$$
\begin{aligned}
S= & \int \frac{d E}{T}=\int \frac{d \mu}{T(\mu)} \frac{d E}{d \mu} \\
= & \frac{\pi \mu^{2}}{G}-2 \pi N\left\{-\frac{L}{\mu}+\left\{-\frac{A}{12}-a+\frac{B}{2}\right) \ln \frac{\mu}{l}+\frac{A}{12}\left(\ln \frac{\mu}{L}\right)^{2}+c\right\} \\
& \left.+\mathcal{O}\left(L^{-1}\right)+\mathcal{O}(G N)^{2}\right) .
\end{aligned}
$$

Here $c$ is the constant of the integration. The classical part coincides with the usual Bekenstein-Hawking entropy when we regard the black hole as the four dimensional object.

Hence we calculated quantum corrections to simplest black hole thermodynamical quantities. The result is actually obtained for two objects: $4 \mathrm{~d} \mathrm{BH}$ and the same object described as $2 \mathrm{~d}$ dilatonic $\mathrm{BH}$. That shows remarkable property of s-wave EA that it could be applied to $4 \mathrm{~d}$ as well as to $2 \mathrm{~d}$ ge-

ometry (where it looks already as complete EA). It is straitforward now to extend the discussion for other types of BHs.

\section{Quantum corrections to SdS and SAdS black holes}

We now consider more general Schwarzschild-(anti-)de Sitter black holes. In the classical limit $(N \rightarrow 0)$ in $(12) \sim(15)$ with non-vanishing cosmological 
constant $\Lambda$, we obtain the usual Schwarzschild-(anti)de Sitter as solution of equations of motion

$$
\mathrm{e}^{2 \rho}=\mathrm{e}^{-2 \sigma}=\mathrm{e}^{2 \rho_{0}} \equiv 1-\frac{\mu}{x}-\frac{\Lambda}{3} x^{2}=-\frac{\Lambda}{3 x} \prod_{i=1}^{3}\left(x-x_{i}\right) .
$$

Here $\mu$ is a constant of the integration corresponding to the black hole mass $\left(\mu=2 G M_{\mathrm{BH}}\right)$. The parameters $x_{i}(i=1,2,3)$ are solutions of the equation $\mathrm{e}^{2 \rho_{0}}=0$. Among $x_{i}$ 's, two are real and positive if $\Lambda>0$ and $\mu^{2}<\frac{4}{9 \Lambda}$ and they correspond to black hole and cosmological horizons in the Schwarzschild-de Sitter black hole. On the other hand, only one is real if $\Lambda<0$. The explicit form of $x_{i}$ is given by

$$
\begin{aligned}
& x_{i}=\left(\alpha_{+}\right)^{\frac{1}{3}}+\left(\alpha_{+}\right)^{\frac{1}{3}}, \quad \omega\left(\alpha_{+}\right)^{\frac{1}{3}}+\omega^{-1}\left(\alpha_{+}\right)^{\frac{1}{3}}, \quad \omega^{-1}\left(\alpha_{+}\right)^{\frac{1}{3}}+\omega\left(\alpha_{+}\right)^{\frac{1}{3}}, \\
& \omega=\mathrm{e}^{\frac{2 i \pi}{3}}, \quad \alpha_{ \pm}=\frac{1}{2 \Lambda}\left(-3 \mu \pm \sqrt{9 \mu^{2}-\frac{4}{\Lambda}}\right) .
\end{aligned}
$$

We should also note that the solutions $\left\{x_{i}, i=1,2,3\right\}$ satisfy the following relations:

$$
\begin{aligned}
& X_{1} \equiv \sum_{i=1}^{3} x_{i}=0 \\
& X_{2} \equiv \sum_{i, j=1, i>j}^{3} x_{i} x_{j}=-\frac{3}{\Lambda}, \quad X_{3} \equiv \prod_{i=1}^{3} x_{i}=-\frac{3 \mu}{\Lambda}
\end{aligned}
$$

If we start from the reduced action (3), the classical solution (47) can be regarded to express the purely two dimensional Schwarzschild-(anti) de Sitter black holes with dilaton. Hence, we again obtain $2 \mathrm{~d}$ or $4 \mathrm{~d}$ formulation for such object. Note that reduction of SAdS BH maybe understood as $2 \mathrm{~d}$ dilatonic $\mathrm{AdS} \mathrm{BH}$ where quantum effects of dilaton were recently discussed in ref. [27.

As in (17), we consider the quantum corrections regarding $G N$ as small. Substituting the classical solution (47) into (18) and integrating it, we obtain

$$
\begin{aligned}
& \Delta_{\rho}+\Delta_{\sigma} \\
& =\Delta_{0}+A\left\{\frac{1}{8 x^{2}}-\frac{1}{12} \sum_{i=1}^{3}\left(\frac{1}{x_{i}\left(x-x_{i}\right)}-\frac{1}{x_{i}^{2}} \ln \left(x-x_{i}\right)\right.\right.
\end{aligned}
$$




$$
\begin{aligned}
& \left.\left.+\frac{1}{x_{i}^{2}} \ln x-\frac{2}{x_{i} x}\right)+\frac{1}{6} \sum_{i=1}^{3} \frac{1}{x_{i} Y_{i}} \ln \left(x-x_{i}\right)\right\} \\
& +\frac{a^{\prime}+B-1}{2 x^{2}}-\frac{1}{2 x^{2}}\left(\ln x+\frac{1}{2}\right) \\
& +\sum_{i=1}^{3}\left\{\frac{1}{2}\left(\frac{1}{x^{2}}-\frac{1}{x_{i}^{2}}\right) \ln \left(x-x_{i}\right)+\frac{1}{2 x_{i}^{2}} \ln x-\frac{1}{2 x_{i} x}\right\} \\
& +\frac{36 t_{0}}{\Lambda^{2}} \sum_{i=1}^{3}\left\{-\frac{1}{x_{i} Y_{i}^{2}} \frac{1}{x-x_{i}}\right. \\
& \left.+\frac{1}{x_{i}^{3} Y_{i}^{3}}\left(4 x_{i}^{2}-\prod_{k=1, k \neq i}^{3} x_{k}\right) \ln \left(x-x_{i}\right)\right\} .
\end{aligned}
$$

Here $\Delta_{0}$ is a constant of the integration and

$$
Y_{i} \equiv \frac{1}{x_{i}} \prod_{i, j=1, i \neq j}^{3}\left(x_{j}-x_{i}\right), \quad a^{\prime} \equiv \ln \left(-\frac{\Lambda}{3}\right)+a .
$$

On the other hand, substituting (47) into (23), one gets

$$
\begin{aligned}
\Delta_{\sigma}= & \frac{1}{\prod_{i=1}^{3}\left(x-x_{i}\right)} \\
& \times\left[\Delta_{1}+A\left\{\frac{x}{6}-\frac{X_{2}}{24 x}+\frac{7 X_{3}}{48 x^{2}}-\frac{1}{24} \sum_{i=1}^{3} Y_{i} \ln \left(1-\frac{x_{i}}{x}\right)\right\}\right. \\
& +\frac{a^{\prime}-B+1}{2}\left(x-\frac{X_{2}}{x}+\frac{X_{3}}{2 x^{2}}\right)+B\left(x+\frac{X_{3}}{4 x^{2}}\right) \\
& -\frac{x}{2}(\ln x-1)+\frac{X_{2}}{2 x}(\ln x+1) \\
& -\frac{X_{3}}{4 x^{2}}\left(\ln x+\frac{1}{2}\right)+\frac{1}{2} \sum_{i=1}^{3}\left(x-x_{i}\right)\left(\ln \left(x-x_{i}\right)-1\right) \\
& +\frac{X_{2}}{2} \sum_{i=1}^{3}\left(-\frac{1}{x} \ln \left(x-x_{i}\right)+\frac{1}{x_{i}} \ln \left(1-\frac{x_{i}}{x}\right)\right) \\
& -\frac{X_{3}}{2}\left(-\frac{1}{2 x^{2}} \ln \left(x-x_{i}\right)+\frac{1}{2 x_{i}^{2}} \ln \left(1-\frac{x_{i}}{x}\right)+\frac{1}{2 x_{i} x}\right) \\
& \left.-\frac{18 t_{0}}{\Lambda^{2}} \sum_{i=1}^{3} \frac{1}{Y_{i}} \ln \left(x-x_{i}\right)\right] .
\end{aligned}
$$


Here $\Delta_{1}$ is a constant of the integration.

Let $x=x_{i=I}$ corresponds to a horizon. Then using (52), near the horizon $x \sim x_{I}$, we find

$$
\begin{aligned}
\Delta_{\sigma} \sim & -\frac{1}{x_{I} Y_{I}} \frac{1}{x-x_{I}}\left[\left\{-\frac{A}{24} Y_{I}+\frac{18 t_{0}}{\Lambda^{2}} \frac{1}{Y_{I}}\right\} \ln \left(x-x_{I}\right)\right. \\
& + \text { regular terms }] .
\end{aligned}
$$

The singularity coming from $\ln \left(x-x_{I}\right)$ vanishes if we choose the parameter $t_{0}$ to be

$$
t_{0}=-\frac{A \Lambda^{2} Y_{I}^{2}}{2^{4} \cdot 3^{3}}
$$

Note that we can remove the singularity corresponding to only one horizon since $Y_{i} \neq Y_{j}$ in general if $i \neq j$. Therefore, in case of Schwarzschild de-Sitter black hole, we cannot remove the singularities corresponding to both of black hole and cosmological horizons.

Using (50) and (54), when $x \sim x_{I}$, we find $\Delta_{\rho}+\Delta_{\sigma}$ is regular

$$
\begin{aligned}
& \Delta_{\rho}+\Delta_{\sigma} \sim B_{I} \\
B_{I} \equiv & \Delta_{0}+A\left[\frac{7}{24 x_{I}^{2}}-\frac{1}{12} \sum_{i=1, i \neq I}^{3}\left\{\left(1-\frac{Y_{I}^{2}}{Y_{i}^{2}}\right)\left(\frac{1}{x_{i}\left(x_{I}-x_{i}\right)}-\frac{1}{x_{i}^{2}} \ln \left(x_{I}-x_{i}\right)\right)\right.\right. \\
& \left.\left.+\frac{1}{x_{i}^{2}} \ln x_{I}-\frac{2}{x_{i} x_{I}}-\frac{1}{6}\left(\sum_{j \neq i, j=1}^{3} \frac{1}{x_{i}\left(x_{i}-x_{j}\right)}\right) \ln \left(x_{I}-x_{i}\right)\right\}-\frac{1}{12 x_{I}^{2}} \ln x_{I}\right] \\
& +\frac{a^{\prime}+B-1}{2 x_{I}^{2}}-\frac{3}{4 x_{I}^{2}} \\
& +\sum_{i=1, i \neq I}^{3}\left\{\frac{1}{2}\left(\frac{1}{x_{I}^{2}}-\frac{1}{x_{i}^{2}}\right) \ln \left(x_{I}-x_{i}\right)+\frac{1}{2 x_{i}^{2}} \ln x_{I}-\frac{1}{2 x_{i} x_{I}}\right\} .
\end{aligned}
$$

Then using (52) and (54), we find

$$
\begin{aligned}
\Delta_{\rho} & \sim-\Delta_{\sigma} \\
& \sim \frac{C_{I}}{x-x_{I}}+\text { regular terms } \\
C_{I} & \equiv \frac{1}{\prod_{i=1, i \neq I}^{3}\left(x-x_{i}\right)}
\end{aligned}
$$




$$
\begin{aligned}
& \times\left[\Delta_{1}+A\left\{\frac{x_{I}}{6}-\frac{X_{2}}{24 x_{I}}+\frac{7 X_{3}}{48 x_{I}^{2}}-\frac{1}{24} \sum_{i=1, i \neq I}^{3}\left(Y_{i}-\frac{Y_{I}^{2}}{Y_{i}}\right) \ln \left(1-\frac{x_{i}}{x_{I}}\right)\right\}\right. \\
& +\frac{a^{\prime}-B+1}{2}\left(x_{I}-\frac{X_{2}}{x_{I}}+\frac{X_{3}}{2 x_{I}^{2}}\right)+B\left(x_{I}+\frac{X_{3}}{4 x_{I}^{2}}\right) \\
& -\frac{x_{I}}{2}\left(\ln x_{I}-1\right)+\frac{X_{3}}{4}\left(\ln x_{I}\right)^{2}+\frac{X_{2}}{2 x_{I}}\left(\ln x_{I}+1\right) \\
& -\frac{X_{3}}{4 x_{I}^{2}}\left(\ln x_{I}+\frac{1}{2}\right)+\frac{1}{2} \sum_{i=1, i \neq I}^{3}\left(x_{I}-x_{i}\right)\left(\ln \left(x_{I}-x_{i}\right)-1\right) \\
& +\frac{X_{2}}{2} \sum_{i=1, i \neq I}^{3}\left(-\frac{1}{x_{I}} \ln \left(x_{I}-x_{i}\right)+\frac{1}{x_{i}} \ln \left(1-\frac{x_{i}}{x_{I}}\right)\right)-\frac{X_{2}}{2 x_{I}} \ln x_{I} \\
& -\frac{X_{3}}{2}\left(-\frac{1}{2 x_{I}^{2}} \ln \left(x_{I}-x_{i}\right)+\frac{1}{2 x_{i}^{2}} \ln \left(1-\frac{x_{i}}{x_{I}}\right)+\frac{1}{2 x_{i} x_{I}}\right) \\
& \left.-\frac{X_{3}}{2}\left(-\frac{1}{2 x_{I}^{2}} \ln x_{I}+\frac{1}{2 x_{I}^{2}}\right)\right] .
\end{aligned}
$$

Eq.(56) tells that the scalar curvature in (33) is regular when $x \sim x_{I}$. The horizon defined by $\mathrm{e}^{2 \rho}=0$, which corresponds to $x=x_{I}$ in the classical limit is given by

$$
\begin{aligned}
0= & \mathrm{e}^{2 \rho} \\
\sim & \mathrm{e}^{2 \rho_{0}}\left(1+2 G N \Delta_{\rho}\right) \\
\sim & -\frac{\Lambda}{3}\left(\prod_{j=1, j \neq I}^{3}\left(x_{j}-x_{I}\right)\right) \\
& \times\left(x_{I}-2 G N C_{I}\right)^{-1}\left(x-x_{I}+2 G N C_{I}\right) .
\end{aligned}
$$

Then the entropy, which is defined by the area of horizon divided by 4 , is given by

$$
\begin{aligned}
S & \sim \pi\left(x_{I}-2 G N C_{I}\right)^{2} \\
& \sim \pi x_{I}^{2}-4 \pi G N C_{I} x_{I} .
\end{aligned}
$$

The second term in (58) corresponds to the quantum correction. When we regard the black holes as purely two dimensional ones starting from (3), it is rather difficult to define the entropy since horizon is a point and does 
not have an area. In case of Schwarzschild de Sitter black holes, it is also difficult to define the entropy in the same way as in Schwarzschild case in (46). The difficulty comes since we cannot define the black hole mass as in (45), where we need to choose the radius $L$ of the universe large, which non-trivial cosmological horizon prevents.

The equation (57) tells that the behavior of the metric near the horizon $x=x_{I}-2 G N C_{I}$ is given by

$$
\begin{aligned}
d s^{2}= & -\mathrm{e}^{2 \rho} d t^{2}+\mathrm{e}^{-2 \rho+2 G N\left(\Delta_{\rho}+\Delta_{\sigma}\right)} d x^{2}+x^{2} d \Omega^{2} \\
\mathrm{e}^{2 \rho} \sim & -\frac{\Lambda}{3}\left(\prod_{j=1, j \neq I}^{3}\left(x_{j}-x_{I}+2 G N C_{I}\right)\right) \\
& \times\left(x_{I}-2 G N C_{I}\right)^{-1}\left(x-x_{I}+2 G N C_{I}\right) \\
\sim & -\frac{\Lambda}{3}\left\{Y_{I}+\left(-6+\frac{2 Y_{I}}{x_{I}}\right) G N C_{I}\right\} \\
& \times\left(x-x_{I}+2 G N C_{I}\right) .
\end{aligned}
$$

Therefore the temperature $T$ is given by

$$
T \sim\left|\frac{\Lambda}{12 \pi}\left\{Y_{I}+\left(-6+\frac{2 Y_{I}}{x_{I}}\right) G N C_{I}-G N Y_{I} B_{I}\right\}\right| .
$$

The second and third terms correspond to the quantum correction. The definition of the temperature is the same if we regard the black holes as purely two dimensional ones. We should note that there are two different temperatures corresponding to the black hole and the cosmological horizons, respectively, for Schwarzschild de Sitter black holes.

The Schwarzschild black hole in the previous section can be obtained as a limit of $\Lambda \rightarrow+0$ in Schwarzschild-de Sitter black hole. In the limit, $x_{i}$ 's in (47) behave as

$$
x_{i} \rightarrow \mu, \pm \sqrt{\frac{3}{\Lambda}} .
$$

Here the constant of the integration $l$ is related with $\Delta_{0}$ by $\Delta_{0}=\frac{1}{12 \mu^{2}} \ln l$ and $\Delta_{S}$ is given by $\Delta_{S}=\Lambda \Delta_{1}+$ const. The additional constant diverges in the limiting procedure $\Lambda \rightarrow+0$ but the divergence can be absorbed into the redefinition of $\Delta_{S}$. 
We now consider de Sitter space as a limit of $\mu \rightarrow 0$. If we choose $x_{I}$ as a cosmological horizon

$$
x_{I}=h \equiv \sqrt{\frac{3}{\Lambda}},
$$

$t_{0}$ in (54) has the following form:

$$
t_{0}=-\frac{A \Lambda}{36}=-\frac{A}{12 h^{2}} .
$$

Then we obtain

$$
\begin{aligned}
& \Delta_{\rho}+\Delta_{\sigma} \\
& =\tilde{\Delta}_{0}-\frac{A}{6 x^{2}}+\frac{a+B-1}{2 x^{2}} \\
& +\frac{1}{2}\left(\frac{1}{x^{2}}-\frac{1}{h^{2}}\right) \ln \left(1-\frac{x^{2}}{h^{2}}\right)+\frac{1}{h^{2}} \ln \frac{x}{h} \\
& \Delta_{\sigma} \\
& =\frac{1}{x\left(x^{2}-h^{2}\right)}\left\{\tilde{\Delta}_{1}+\frac{A x}{6}+\frac{a-B+1}{2}\left(x+\frac{h^{2}}{x}\right)\right. \\
& \left.+B x+\frac{(x-h)^{2}}{2 x} \ln \left(1-\frac{x}{h}\right)+\frac{(x+h)^{2}}{2 x} \ln \left(1+\frac{x}{h}\right)\right\} .
\end{aligned}
$$

Here the constants $\tilde{\Delta}_{0}$ and $\tilde{\Delta}_{1}$ are related with the constants of the integration $\Delta_{0}$ and $\Delta_{1}$ by

$$
\tilde{\Delta}_{0}=\Delta_{0}+\frac{1}{2 h^{2}} \ln (-1), \quad \tilde{\Delta}_{1}=\Delta_{1}+h .
$$

The expressions (64) and (65) can be used for anti-de Sitter space by analytically continuing $h$ by $h \rightarrow i \sqrt{-\frac{3}{\Lambda}}$ :

$$
\begin{aligned}
& \Delta_{\rho}+\Delta_{\sigma} \\
& =\tilde{\Delta}_{0}-\frac{A}{6 x^{2}}+\frac{a+B-1}{2 x^{2}} \\
& +\frac{1}{2}\left(\frac{1}{x^{2}}+\frac{1}{h^{2}}\right) \ln \left(1+\frac{x^{2}}{h^{2}}\right)-\frac{1}{h^{2}} \ln \frac{x}{h} \\
& \Delta_{\sigma}
\end{aligned}
$$




$$
\begin{aligned}
& =\frac{1}{x\left(x^{2}+h^{2}\right)}\left\{\tilde{\Delta}_{1}+\frac{A x}{6}+\frac{a-B+1}{2}\left(x-\frac{h^{2}}{x}\right)\right. \\
& \left.+B x+\frac{x^{2}-h^{2}}{2 x} \ln \left(1+\frac{x^{2}}{h^{2}}\right)+2 h \arctan \left(\frac{x}{h}\right)\right\} .
\end{aligned}
$$

We should also note that there appears a singularity at the origin $x=0$, which might be a sign that the $s$-wave approximation is not valid there since the origin is always a singularity of $s$-wave. The $s$-wave approximation, however, should be valid near the cosmological horizon.

Near the classical horizon $x \sim h$, we find

$$
\begin{aligned}
\Delta_{\rho}+\Delta_{\sigma} & \sim \tilde{B} \\
\tilde{B} & \equiv \tilde{\Delta}_{0}-\frac{A}{6 h^{2}}+\frac{a+B-1}{2 h^{2}} \\
\Delta_{\sigma} & \sim \frac{\tilde{C}}{x-h} \\
\tilde{C} & \equiv \frac{\tilde{\Delta}_{1}+\frac{A h}{6}+(a+1+2 \ln 2) h}{2 h^{2}} .
\end{aligned}
$$

Then putting $x_{I}=h$ and $C_{I}=\tilde{C}$ in (58), we find the expression for the entropy $S$ :

$$
\begin{aligned}
S & =\pi h^{2}-4 \pi G N \tilde{C} h \\
& =\frac{3 \pi}{\Lambda}-2 \pi G N\left\{\tilde{\Delta}_{1} \sqrt{\frac{\Lambda}{3}}+\frac{A}{6}+a+1+2 \ln 2\right\} .
\end{aligned}
$$

It is interesting to note that last expression describes quantum corrections to the entropy of expanding inflationary Universe (as de Sitter space may be considered as such inflationary Universe). That gives new terms proportional to particles number as compare with classical entropy of expanding Universe discussed extensively in refs. 21.

Taking $Y_{I}=2 h$ and $B_{I}=\widetilde{B}$ in (60), the temperature is expressed as

$$
\begin{aligned}
T & =\frac{\Lambda}{12 \pi}\{2 h+G N(2 \tilde{C}-2 h \tilde{B})\} \\
& =\frac{1}{2 \pi} \sqrt{\frac{\Lambda}{3}}+\frac{G N \Lambda}{12 \pi}\left\{\frac{\Lambda \tilde{\Delta}_{1}}{3}-2 \tilde{\Delta}_{0} \sqrt{\frac{3}{\Lambda}}+\left(\frac{A}{2}+2+2 \ln 2-B\right) \sqrt{\frac{\Lambda}{3}}\right\} .
\end{aligned}
$$


Hence we found quantum corrections to the temperature and the entropy for $4 \mathrm{~d}$ de Sitter space (as the limit of SdS BH) as well as for SdS BH and SAdS $\mathrm{BH}$. In the last case of SdS or SAdS BHs we also defined the quantum correction to the temperature of corresponding $2 \mathrm{~d}$ object (i.e. corresponding $\mathrm{BH}$ with dilaton). The calculation of $2 \mathrm{~d}$ quantum entropy is more difficult and cannot be done by using only $4 \mathrm{~d}$ point of view unlike the case of Schwarzschild $\mathrm{BH}$.

\section{Quantum corrections to 't Hooft BH model}

We now consider 't Hooft's BH model [22]. In the model, the Hawking massless particles emitted from a black hole are treated as an envelope of matter which obeys the classical equation of state, and acts as a source of the gravity. Then, the horizon vanishes and the black hole entropy can be calculated as that of the particles by using the Hartree-Fock approximation. In this model $\mathrm{BH}$ entropy calculated with such recipe does not give the standard $1 / 4$ coefficient in area law. Below we only consider scalars for simplicity, then one can put $A=B=1$ in formulas of previous section.

In the Rindler space limit, where the black hole mass $M_{\mathrm{BH}}=\frac{\mu}{2 G}$ is large, the metric has the following form 22

$$
\mathrm{e}^{2 \rho_{0}}=\frac{\lambda^{2}}{P}\left(\frac{2 M_{\mathrm{BH}}}{x}\right)^{6}, \quad \mathrm{e}^{2 \sigma_{0}}=\frac{\lambda^{2}}{P^{3}}\left(\frac{2 M_{\mathrm{BH}}}{x}\right)^{14},
$$

where

$$
P \equiv \frac{1}{5}\left\{\left(\frac{2 M_{\mathrm{BH}}}{x}\right)^{5}-1\right\}, \lambda \equiv \frac{1}{48 M_{\mathrm{BH}}} \sqrt{\frac{N}{5 \pi}} .
$$

In the following, we put $G=1$ when there is no any confusion. Following [22], a new coordinate $y$ is introduced by

$$
y \equiv\left(\frac{2 M_{\mathrm{BH}}}{x}\right)^{5}
$$

We suppose that matter quantum effects are described by the same effective action as in section 2 (without spinors). Then one can use the same effective action as quantum correction to 't Hooft equations of motion. That, of course, adds new terms to these equations. 
To be more specific, substituting the classical solution (73) into (18) and subtracting the contribution from the classical matter, we obtain

$$
\begin{aligned}
& \Delta_{\rho}+\Delta_{\sigma} \\
& =\frac{1}{\left(2 M_{\mathrm{BH}}\right)^{2}}\left[\Delta_{0}^{\prime}-2 y^{\frac{2}{5}}+\frac{3}{5} F_{\frac{2}{5}}(y)+\frac{5}{12} \frac{y^{\frac{7}{5}}}{y-1}\right. \\
& +\frac{3}{5} y^{\frac{2}{5}}\left(\ln y-\frac{5}{2}\right)-\frac{1}{2} y^{\frac{2}{5}} \ln (y-1)+\frac{a_{1}}{2} y^{\frac{2}{5}} \\
& \left.-80 M_{\mathrm{BH}}^{2} t_{0}\left(-\frac{y^{\frac{3}{5}}}{y-1}+\frac{3}{5} F_{-\frac{2}{5}}(y)\right)\right] .
\end{aligned}
$$

Here $\Delta_{0}^{\prime}$ is a constant of the integration and a constant $a_{1}$ is defined by

$$
a_{1} \equiv a+\ln \left(5 \lambda^{2}\right) \text {. }
$$

We also introduced a function $F_{\alpha}(y)$ which is defined by

$$
F_{\alpha}(y) \equiv \sum_{n=0}^{\infty} \frac{1}{\alpha-n} y^{\alpha-n},
$$

which appears in the integration of $\int^{y} d y \frac{y^{\alpha}}{y-1}$. The constants of the integration are always absorbed into the definition of $\Delta_{0}^{\prime}$ in $(76)$ (and $\Delta_{1}^{\prime}$ in the later eq. (83)).

When $y \sim 1$, we find the behavior of $\Delta_{\rho}+\Delta_{\sigma}$ from $(78)$

$$
\Delta_{\rho}+\Delta_{\sigma} \sim\left(\frac{5 A}{12}+80 M_{\mathrm{BH}}^{2} t_{0}\right) \frac{1}{y-1} .
$$

Requiring that the singularity vanishes, we can determine the constant $t_{0}$ by

$$
t_{0}=-\frac{1}{2^{6} \cdot 3 M_{\mathrm{BH}}^{2}} \text {. }
$$

On the other hand, substituting the classical solution (73) into (23) (and subtracting the contribution from the classical matter again), we obtain

$$
\begin{aligned}
\Delta_{\sigma}= & \frac{\mathrm{e}^{-G(y)}}{\left(2 M_{\mathrm{BH}}\right)^{2}} \int^{y} d y \mathrm{e}^{G(y)}\left[-\frac{23}{10} y^{-\frac{3}{5}}+\frac{8}{3} \frac{y^{\frac{2}{5}}}{y-1}-\frac{5}{24} \frac{y^{\frac{7}{5}}}{(y-1)^{2}}\right. \\
& \left.+\left(\frac{3}{25} \ln y-\frac{1}{10} \ln (y-1)+\frac{a_{2}}{10}\right) y^{-\frac{3}{5}}-40 M_{\mathrm{BH}}^{2} t_{0} \frac{y^{\frac{3}{5}}}{(y-1)^{2}}\right]
\end{aligned}
$$


Here

$$
\begin{aligned}
G(y) \equiv & -25 \lambda^{2}\left\{-\frac{y^{\frac{9}{5}}}{2(y-1)^{2}}-\frac{9}{10} \frac{y^{\frac{4}{5}}}{y-1}+\frac{18}{25} F_{-\frac{1}{5}}(y)\right\} \\
= & -25 \lambda^{2}\left[-\frac{y^{\frac{9}{5}}}{2(y-1)^{2}}-\frac{9}{10} \frac{y^{\frac{4}{5}}}{y-1}\right. \\
& +\frac{18}{25}\left\{\sqrt{\frac{5+\sqrt{5}}{2}} \tan ^{-1}\left(2 \sqrt{\frac{2}{5+\sqrt{5}}}\left(\frac{1-\sqrt{5}}{4}+y^{\frac{1}{5}}\right)\right)\right. \\
& +\sqrt{\frac{5-\sqrt{5}}{2}} \tan ^{-1}\left(2 \sqrt{\frac{2}{5-\sqrt{5}}}\left(\frac{1+\sqrt{5}}{4}+y^{\frac{1}{5}}\right)\right)+\ln \left(-1+y^{\frac{1}{5}}\right) \\
& -\frac{1-\sqrt{5}}{4} \ln \left(1+\frac{1-\sqrt{5}}{2} y^{\frac{1}{5}}+y^{\frac{2}{5}}\right) \\
& \left.-\frac{1+\sqrt{5}}{4} \ln \left(1+\frac{1+\sqrt{5}}{2} y^{\frac{1}{5}}+y^{\frac{2}{5}}\right)\right\}
\end{aligned}
$$

In the limit of $M_{\mathrm{BH}} \rightarrow \infty, G(y)$ vanishes and we obtain

$$
\begin{aligned}
\Delta_{\sigma}= & \frac{1}{\left(2 M_{\mathrm{BH}}\right)^{2}}\left\{\Delta_{1}^{\prime}-\frac{13}{2} y^{\frac{2}{5}}+\frac{5}{24} \frac{y^{\frac{7}{5}}}{y-1}+\frac{21}{8} F_{\frac{2}{5}}(y)+\frac{3}{10} y^{\frac{2}{5}} \ln y\right. \\
& \left.-\frac{1}{4} y^{\frac{2}{5}} \ln (y-1)+\frac{1}{4} F_{\frac{2}{5}}(y)+\frac{a_{2}}{4} y^{\frac{2}{5}}-40 M_{\mathrm{BH}}^{2} t_{0}\left(-\frac{y^{\frac{3}{5}}}{y-1}+\frac{3}{5} F_{-\frac{2}{5}}(y)\right)\right\} .
\end{aligned}
$$

Here $\Delta_{1}^{\prime}$ is a constant of the integration. If we choose $t_{0}$ by (80), Eqs. (76) and (83) tell

$$
\Delta_{\rho}, \quad \Delta_{\sigma}=\mathcal{O}(\ln (y-1)) \quad \text { when } y \rightarrow 1+0
$$

We also find

$$
\Delta_{\rho}, \quad \Delta_{\sigma}=\mathcal{O}\left(y^{\frac{2}{5}}\right) \quad \text { when } y \rightarrow+\infty .
$$

Using (76) and (81), we can estimate the entropy with the help of the expression in 22] for massless scalar fields :

$$
S=\frac{2 \pi^{2} \Sigma}{45 \beta^{3}} \int d y \frac{H(y) \sqrt{F(y)}}{A(y)^{\frac{3}{2}}} .
$$


Here $\beta=\frac{1}{T}$ and the metric is assumed to have the following form

$$
d s^{2}=-A(y) d t^{2}+F(y) d y^{2}+\left(2 M_{\mathrm{BH}}\right)^{2} H(y) d \Omega^{2}
$$

and $\Sigma$ is the area of the surface given by $y=1$, i.e., $\Sigma=4 \pi\left(2 M_{\mathrm{BH}}\right)^{2}$. Eq. (866) expresses the contribution from one kind of massless scalar field. Then total entropy is given by multiplying $N$ with $S$ in (86). We now find the following expression including the quantum correction

$$
S=\frac{2 \pi^{2} \Sigma}{45 \beta^{3}} \frac{2 M_{\mathrm{BH}}}{5 \lambda^{2}} \int_{1}^{\infty} \frac{d y}{y^{2}}\left(1+N\left(\Delta_{\sigma}-3 \Delta_{\rho}\right)\right) .
$$

Substituting (76) and (83) into (88), we obtain

$$
\begin{aligned}
S= & \frac{2 \pi^{2} \Sigma}{45 \beta^{3}} \frac{2 M_{\mathrm{BH}}}{5 \lambda^{2}} \int_{1}^{\infty} \frac{d y}{y^{2}}\left[1+\frac{N}{\left(2 M_{\mathrm{BH}}\right)^{2}}\left\{-3 \Delta_{0}^{\prime}+4 \Delta_{1}^{\prime}\right.\right. \\
& +2 F_{\frac{1}{2}}(y)-\frac{5}{12} \frac{y^{\frac{7}{5}}}{y-1}-\frac{3}{5} y^{\frac{2}{5}} \ln y-\frac{31}{2} y^{\frac{2}{5}} \\
& +\frac{1}{2} y^{\frac{2}{5}} \ln (y-1)+\frac{43}{10} F_{\frac{2}{5}}(y)+\left(-\frac{3}{2} a_{1}+a_{2}\right) y^{\frac{2}{5}} \\
& \left.\left.-80 M_{\mathrm{BH}}^{2} t_{0}\left(\frac{y^{\frac{3}{5}}}{y-1}-\frac{3}{5} F_{-\frac{2}{5}}(y)\right)\right\}\right] .
\end{aligned}
$$

Using (80) and the following numerical results:

$$
\begin{aligned}
& \int_{1}^{\infty} \frac{F_{\frac{2}{5}}(y)}{y^{2}} d y=-\int_{1}^{\infty} \frac{F_{-\frac{2}{5}}(y)}{y^{2}} d y=\frac{5}{2}=2.5 \cdots \\
& \int_{1}^{\infty} \frac{y^{-\frac{3}{5}}-y^{-\frac{7}{5}}}{y-1} d y=1.47923 \cdots \\
& \int_{1}^{\infty} y^{-\frac{8}{5}} \ln (y-1) d y=1.60567 \cdots
\end{aligned}
$$

we get

$$
\begin{aligned}
S= & \frac{2 \pi^{2} \Sigma}{45 \beta^{3}} \frac{2 M_{\mathrm{BH}}}{5 \lambda^{2}}\left[1+\frac{N}{\left(2 M_{\mathrm{BH}}\right)^{2}}\left\{-3 \Delta_{0}^{\prime}+4 \Delta_{1}^{\prime}-10.93851\right.\right. \\
& \left.\left.+1.666667\left(-\frac{3}{2} a_{1}+a_{2}\right)\right\}\right] .
\end{aligned}
$$


In [22], the entropy comes from the massless scalar fields in the background including the backreaction by Hawking radiation. The obtained entropy is different from the Bekenstein-Hawking entropy by a factor $\frac{8}{5}$ at the classical level. Note that there are another examples of BHs (like Kerr-bolt-AdS) where there are deviations from Bekenstein-Hawking law. In (91), the correction to the entropy is estimated. However, as quantum corrections are assumed to be small perturbation it was hard from the very beginning to expect that they may change the qualitative structure of the model. In particular, BH entropy does not reproduce the Bekenstein-Hawking law.

If we assume $\frac{1}{T}=\beta=8 \pi M_{\mathrm{BH}}$, the thermodynamical mass defined by (38) can be given by

$$
\begin{aligned}
E= & \int d T T \frac{d S}{d T} \\
= & \frac{2}{5 N} \frac{1}{4 \pi T}\left[2+\frac{(4 \pi)^{2} N}{2} T^{3}\left\{-3 \Delta_{0}^{\prime}+4 \Delta_{1}^{\prime}-10.93851\right.\right. \\
& \left.+1.666667\left(-\frac{3}{2} a_{1}+a_{2}\right)\right\} \\
= & \frac{4 M_{\mathrm{BH}}}{5 N}\left[2+\frac{N}{64 M_{\mathrm{BH}}^{3}}\left\{-3 \Delta_{0}^{\prime}+4 \Delta_{1}^{\prime}-10.93851\right.\right. \\
& \left.+1.666667\left(-\frac{3}{2} a_{1}+a_{2}\right)\right\} .
\end{aligned}
$$

The "mass" $M_{\mathrm{BH}}$ is originally the mass of the black hole measured in the asymptotically flat region, i.e., outside of the black hole. In this section, we can regard $M_{\mathrm{BH}}$ is defined by the temperature.

Hence, new methods (not semiclassical ones) are necessary in strong quantum gravity regime in order to solve the problems related with 't Hooft BH

model. It could be that BH-thermodynamical laws in such regime should be completely revised.

\section{Discussion}

We studied quantum properties of $\mathrm{S}(\mathrm{A}) \mathrm{dS}$ BHs using anomaly induced EA for dilaton coupled matter. The explicit evaluation of thermodynamic quantities (temperature, mass, entropy) with account of quantum effects is done for 
S(A)dS BHs as well as for their limiting cases: Schwarzschild and de Sitter spaces. The case of 't Hooft BH model is also considered.

The anomaly induced EA under discussion gives the possibility for $4 \mathrm{~d}$ formulation ( $s$-wave approximation) and for $2 \mathrm{~d}$ formulation. Hence, the corresponding results (with small modifications) are valid for the same background being quantum corrected $4 \mathrm{~d} \mathrm{BH}$ or quantum corrected $2 \mathrm{~d}$ dilatonic $\mathrm{BH}$. The formulation is general enough. There is no problem to apply the same technique for the calculation of quantum corrections to any other gravitational background. Using of other versions of EA (say, the one induced by $4 \mathrm{~d}$ anomaly) does not make big qualitative difference.

Note, however, that we considered quantum EA as small perturbation to classical one. In this way, we estimated quantum corrections to the entropy of de Sitter (inflationary) Universe. Clearly, such investigation should be more important when quantum effects play the dominant role (say, gravitational background is induced by quantum effects). As an example, let us consider $\mathcal{N}=4 S U(N)$ super YM theory. In this case, starting with zero cosmological constant we get the effective cosmological constant [26]: $H^{2}=-\frac{1}{\kappa b^{\prime}}$, $b^{\prime}=-\frac{\left(N^{2}-1\right)}{(8 \pi)^{2}}$ and $\kappa$ is gravitational constant. Hence, classical entropy of de Sitter Universe is quantum entropy on the same time and it is given by the standard expression with the change of cosmological constant by the effective cosmological constant $H^{2}$. It would be really interesting to investigate the questions related with the quantum entropy of BHs and expanding Universe (when there is cosmological horizon [21]) in such strong quantum regime.

Acknowledgments. SDO would like to thank G. Gibbons, E. Mottola and G.'t Hooft for useful discussion of some related questions. The research by SDO was partially supported by a RFBR Grant N 99-02-16617, by Saxonian Min. of Science and Arts and by Graduate College "Quantum Field Theory" at Leipzig University.

\section{References}

[1] J.M. Bardeen, B. Carter and S.W. Hawking, Comm.Math.Phys. 31 (1973) 161. 
[2] J.D. Bekenstein, Nuovo Cim.Lett. 4 (1972) 737; S.W. Hawking, Comm.Math.Phys. (1975) 199.

[3] A. Strominger and C. Vafa, Phys.Lett. B379 (1996) 99; for a review, see A. Peet, Class.Quant.Grav. 15 (1998) 3291.

[4] S.Carlip, hep-th/9806026.

[5] A. Bytsenko, L. Vanzo and S. Zerbini, Phys.Rev. D57 (1998) 4917.

[6] T. Jacobson, gr-qc/9404039.

[7] E. Elizalde, S. Naftulin and S.D. Odintsov, Phys.Rev. D49 (1994) 2852.

[8] R. Bousso and S. Hawking, Phys.Rev. D56 (1997) 7788.

[9] S. Nojiri and S.D. Odintsov, Mod.Phys.Lett. A12 (1997) 2083; Phys.Rev. D57 (1998) 4847.

[10] S. Nojiri and S.D. Odintsov, Phys.Rev. D57 (1998) 2363; T. Chiba and M. Siino, Mod.Phys.Lett. A12 (1997) 709; S. Ichinose, Phys.Rev. D57 (1998) 6224; A. Mikovic and V. Radovanovic, Class.Quant.Grav. 15 (1998) 827; W. Kummer, H.Liebl and D.V. Vassilevich, Mod.Phys.Lett. A12 (1997) 2683; J.S. Dowker, Class.Quant.Grav. 15 (1998) 1881; S. Ichinose and S.D. Odintsov, Nucl.Phys. B539 (1999) 643.

[11] I.L. Buchbinder, S.D. Odintsov and I.L. Shapiro, Effective Action in Quantum Gravity, IOP Publishing, Bristol and Philadelphia, 1992.

[12] S. Nojiri and S.D. Odintsov, hep-th/9806055, Phys.Rev. D59 (1999) 044003;

[13] R. Bousso and S.W. Hawking, Phys.Rev. D57 (1998) 2436; R. Bousso, Phys.Rev. D58 (1998) 083511.

[14] M. Burić, V. Radovanović and A. Miković, gr-qc/9804083.

[15] A.J.M. Medved and G. Kunstatter, hep-th/9904070.

[16] F. Lombardo, F.D. Mazzitelli and J. Russo, Phys. Rev. D59 (1999) 084002; R. Balbinot and A. Fabbri, Phys.Rev. D59 (1999) 044031; 
[17] P. van Nieuwenhuizen, S. Nojiri and S.D. Odintsov, hep-th/9901119.

[18] C. Callan, S. Giddings, J. Harvey and A. Strominger, Phys.Rev. D45 (1992) 1005.

[19] J. Russo, L. Susskind and L. Thorlacius, Phys.Lett. B292 (1992) 13.

[20] S.P. de Alwis, Phys.Lett. B289 (1992) 278; A. Bilal and C. Callan, Nucl.Phys. B394 (1993) 73; S. Nojiri and I. Oda, Phys.Lett. B294 (1992) 317; Nucl.Phys. B406 (1993) 499; T. Banks, A. Dabholkar, M. Douglas and M. O'Loughlin, Phys.Rev. D45 (1992) 3607; R.B. Mann, Phys.Rev. D47 (1993) 4438; D. Louis-Martinez and G. Kunstatter, Phys.Rev. D49 (1994) 5227; J. Polchinski and A. Strominger, Phys.Rev. D50 (1994) 7403; T.Klobsch and T.Strobl, Class.Quant.Grav. 13 (1996) 965; S. Bose, L. Parker and Y. Peleg, Phys.Rev. D52 (1995) 3512; for a review,see A. Strominger, Les Houches lectures on black holes, hepth/9501071.

[21] G. Gibbons, Nucl.Phys. B292 (1987) 784 ; B310 (1988) 636; B472 (1996) 683.

[22] G. 't Hooft, Nucl.Phys.Proc.Suppl. 68 (1998) 174, gr-qc/9706058 and gr-qc/9711053.

[23] R. Bousso and S.W. Hawking, Phys.Rev. D54 (1996) 6312.

[24] A. Bytsenko, S. Nojiri and S.D. Odintsov, Phys.Lett. B443 (1998) 121.

[25] S. Nojiri and S.D. Odintsov, Phys.Rev. D59 (1999) 044026; E. Elizalde, S. Nojiri and S.D. Odintsov, Phys.Rev. D59 (1999) 061501.

[26] I. Brevik and S.D. Odintsov, hep-th/9902184, PLB ,to appear.

[27] W.T. Kim and J. Oh, hep-th/9905007. 